\title{
Omental torsion presenting as acute abdominal pain, pre- operative diagnosis: A case report
}

\author{
Mohammad N Athamnah*, Hussein SO Al Azzam, Raid FF Maayah, Laith MY Maqableh, Ali H Malkawi, Nimah A Rabai, Ali M Zetun, \\ Fayez M Haddad and Mohammad AK Alshloul
}

Department of General Surgery, Princess Basma Teaching Hospital / Jordan Ministry of Health, Irbid Jordan

\begin{abstract}
Omental torsion is a rare cause of acute abdominal pain that is not commonly diagnosed pre-operatively. The increased use of imaging such as computerized tomography in evaluating patients presenting with acute abdominal pain allowed the pre-operative diagnosis of omental torsion to be made much more often. Here we present a case of 18-year-old male who visited our emergency department complaining of right lower abdominal pain for several days. After proper evaluation by clinical examination and imaging, namely CT scan, provisional diagnosis was omental torsion. Differential diagnosis was acute appendicitis. The patient was admitted to the surgical ward. Surgery was performed through a Gridiron incision. Omental torsion with necrosis was found. Diseased omentum was excised and appendectomy performed.
\end{abstract}

\section{Introduction}

Omental torsion is defined as the omentum twisting along its own long axis, compromising blood supply [1]. Omental torsion is not commonly diagnosed before surgery. Awareness of this condition as a differential diagnosis in acute abdominal pain is important to surgeons because it resembles common causes of acute surgical abdomen [2]. Until recently, the diagnosis of Omental torsion was usually made during surgery for a presumed acute appendicitis or peritonitis. As computerized tomography (CT) is becoming standard of care for acute abdominal pain evaluation, before undergoing any surgical intervention, the diagnosis of Omental torsion is being made more often pre-operatively [3]. The best management of pre-operatively diagnosed Omental torsion is controversial. The debate between early surgical treatment (open or laparoscopic) versus conservative management is not settled [4]. Conservative management includes oral analgesics, antiinflammatory drugs, and prophylactic antibiotics $[5,6]$. Conservative management can expose the patient to several complications, such as abscesses formation and intra-abdominal adhesions [6,7]. The strongest and most important indication against conservative management is the possibility of missing the diagnosis of acute appendicitis [4] which can expose patients to unnecessary complications of untreated acute appendicitis. Here, we present a case of Omental torsion, diagnosed before surgery, treated with open surgical excision in an 18 -year-old male.

\section{Case presentation}

A previously healthy 18 -year-old male patient presented to our emergency department complaining of abdominal pain. Pain started 3 days before, mainly in right lower abdomen radiating to epigastric area. Severity of pain was 7/10; pain was vague and dull in nature, increased by movement and food ingestion, relieved by sitting still and fasting. No history of similar pain attacks before, it was the first time the patient experienced such pain. Pain was associated with nausea, anorexia and fever. Patient reported no vomiting and no change in bowel habit. Upon physical exam, the patient was lying in bed in pain. Temperature was $38 \mathrm{C}$ and normal blood pressure. Abdomen was tender to exam, mainly in right iliac fossa with positive rebound tenderness at McBurney's point. Complete blood count showed Haemoglobin of $16 \mathrm{~g} / \mathrm{dl}$, Platelets count of 192.000 and White Blood Cell count of 9.000. Liver and kidney function tests were normal. After CT scan, the diagnosis of Omental torsion was made. Figure 1 shows abdominal CT scan (transverse plane), the hyper-dense peripheral halo and swirling of omental vessels, which suggested a diagnosis of Omental torsion. Figure 2 CT scan (Coronal view) showing area of fat stranding supported the diagnosis of Omental torsion.

Open surgery was performed through Gridiron incision. Necrotic diseased momentum was easily identified and excised. Appendectomy was done, to prevent diagnostic confusion in the future for any lower abdominal pain that may occur. Recovery was smooth, patient improved dramatically after surgery and was discharged home in postoperative day 3 .

\section{Discussion}

Omental torsion is a rare disease, usually diagnosed intra-operatively for suspected acute appendicitis. The incidence of Omental torsion is as rare as $0.37 \%$ of assumed acute appendicitis cases treated by surgery [8]. Increased use of radiological imaging for investigating potential causes of acute abdominal pain allowed more pre-operative identification of Omental torsion [4]. Infarction is the main complication of Omental

${ }^{*}$ Correspondence to: Mohammad Naser Athamnah, Department of General Surgery, Princess Basma Teaching Hospital / Jordan Ministry of Health, Irbid, Jordan, E-mail: mathamneh85@gmail.com

Key words: omental torsion, omental infarction, acute abdominal pain, acute appendicitis

Received: December 15, 2020; Accepted: December 27, 2020; Published: December 30, 2020 


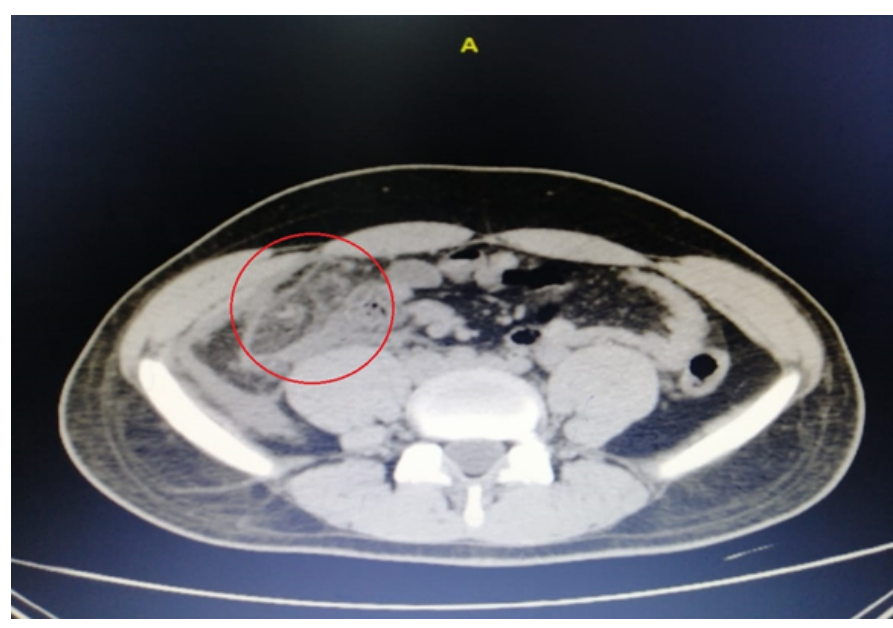

Figure 1. Transverse plane CT scan showing peripheral halo and swirling of omental vessels in right lower abdomen

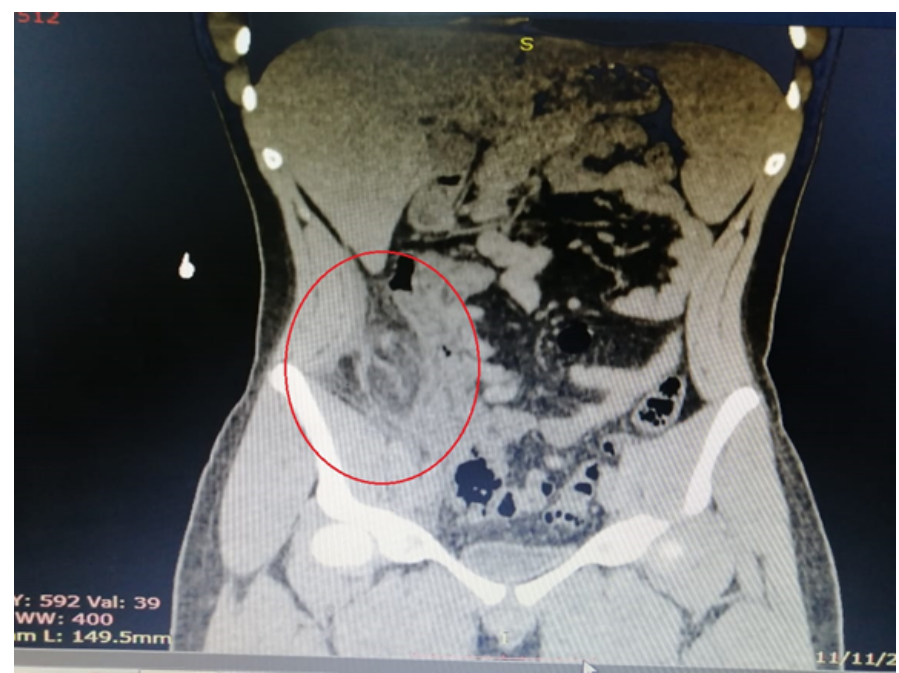

Figure 2. Coronal view CT scan showing peripheral halo and area of fat stranding strongly supporting the diagnosis

torsion. The first described case of Omental torsion leading to infarction was documented by Eitel in 1899 [9]. After that, many case reports and case series reported Omental torsion describing its presentation and management [10].

Omental torsion can be primary or secondary [4]. Accessory omentum, sudden movements, hyper-peristalsis, violent exercise and obesity can cause primary Omental torsion. Secondary omental torsion can happen due to hernia, tumour, cyst or adhesions [4,11]. During surgery for the patient in this case report, the distal end of the omentum was gangrenous with moderate amount of serosanguinous free fluid in the peritoneal cavity. No adhesive bands or torsion leading points were seen. Patient in this case report was diagnosed as primary Omental torsion as no causative agent responsible for a secondary torsion was identified.

Although surgery is the mainstay of Omental torsion treatment, conservative management is suggested in certain cases [4]. The widespread use of imaging modalities for the evaluation of acute abdominal pain allowed more accurate diagnosis of Omental torsion outside the operation room. Conservative treatment of Omental torsion consists of observation, pain management, anti-inflammatory medications and prophylactic antibiotics. Few papers reported successful conservative management of Omental torsion diagnosed and confirmed by CT scan. $[5,6,11,12]$. Complications of conservative management include the possibility of abscess formation and formation of adhesive bands, not to mention the possibility of missing the diagnosis of acute appendicitis and its complications.

In this case report, our patient was diagnosed pre-operatively by CT scan as Omental torsion. Surgery was performed to treat the situation in a decisive and timely manner in order to avoid conservative management complications and also to prevent prolonged hospital stay due to COVID-19 pandemic. Fast recovery is needed because our hospital is heavily affected by Corona virus situation. Long patient hospital stay is not recommended, to prevent Corona virus spread and cross infection also to save supplies and human resources. We decided to operate in a classic open manner (Gridiron incision) rather than laparoscopic technique to avoid longer operative time and to facilitate the extraction of the diseased omentum. The size of diseased omentum could not be evaluated before surgery. Hence, open surgery was the choice because it is difficult to extract sizable tissue through laparoscopic incisions.

The best management of pre-operatively diagnosed Omental torsion is disputable, compromising a research gap. Hopefully, in the future, after Covid-19 pandemic, more patients diagnosed with Omental torsion pre-operatively can be enrolled in clinical studies. More research is needed to compare outcomes of conservative management versus surgical management in terms of hospital stay, morbidity and cost effectiveness.

\section{Conclusion}

Omental torsion is a rare disease, previously diagnosed intraoperatively. The increased use of CT scan for acute abdominal pain evaluation allowed more pre-operative diagnosis of this disease. Omental torsion can be primary or secondary to underlying pathology. Management can be conservative or surgical. Conservative management is not without complications. Surgical management is decisive with faster recovery compared with conservative management. Although diagnosis of Omental torsion was made before surgery, Covid-19 pandemic compelled surgical treatment for our patient, in an effort to prevent prolonged hospital stay.

\section{References}

1. Jain P, Chhabra S, Parikh K, Vaidya A (2008) Omental torsion. J Indian Assoc Pediatr Surg 13: 151. [Crossref]

2. Gaxa L, Elsenbroek C, Jonkers F, Makhanya N, Lockhat Z, et al. (2020) Greater omental torsion secondary to a right inguinal hernia: a case and literature review. World J Surg Surgical Res 3: 1187.

3. Miguel JP, Aguayo JA, Soria VA, Aguilar JJ, Flores BP, et al. (2002) Omental torsion imaging techniques can prevent unnecessary surgical interventions. Gastroenterol Hepatol 25: 493-6. [Crossref]

4. Itenberg E, Mariadason J, Khersonsky J, Wallack M (2010) Modern management of omental torsion and omental infarction: a surgeon's perspective. J Surg Educ 67: 44-7. [Crossref]

5. Coulier B (2006) Segmental omental infarction in childhood: a typical case diagnosed by CT allowing successful conservative treatment. Pediatr Radiol 36: 141-3. [Crossref]

6. Fragoso AC, Pereira JM, Estevão-Costa J (2006) Nonoperative management of omental infarction: a case report in a child. J Pediatr Surg 41: 1777-9. [Crossref]

7. Sánchez J, Rosado R, Ramírez D, Medina P, Mezquita S, et al. (2002) Torsion of the greater omentum: treatment by laparoscopy. Surg Laparosc Endosc Percutan Tech 12: 443-5. [Crossref]

8. Pinedo-Onofre JA, Guevara-Torres L (2007) Omental torsion. An acute abdomen etiology. Gac Med Mex 143: 17-20. [Crossref] 
9. Leitner MJ, Jordan CG, Spinner MH, Reese EC (1952) Torsion, infarction and hemorrhage of the omentum as a cause of acute abdominal distress. Ann surg 135: 103. [Crossref]

10. Medina-Gallardo NA, Curbelo-Peña Y, Stickar T, Gardenyes J, Fernández-Planas S, et al. (2020) Omental infarction: surgical or conservative treatment? A case reports and case series systematic review. Ann Med Surg (Lond) 56: 186-193. [Crossref]
11. Vriesman AB, Lohle PN, Coerkamp EG, Puylaert JB (1999) Infarction of omentum and epiploic appendage: diagnosis, epidemiology and natural history. Eur Radiol 9: 1886-92. [Crossref]

12. Nubi A, McBride W, Stringel G (2009) Primary omental infarct: conservative vs operative management in the era of ultrasound, computerized tomography, and laparoscopy. J Pediatr Surg 44: 953-6.

Copyright: $@ 2020$ Athamnah MN. This is an open-access article distributed under the terms of the Creative Commons Attribution License, which permits unrestricted use, distribution, and reproduction in any medium, provided the original author and source are credited. 